\title{
Local kinetic-energy density of the Airy gas
}

\author{
Vitos, Levente; Johansson, B.; Kollár, J.; Skriver, Hans Lomholt
}

Published in:

Physical Review A

Link to article, DOI:

10.1103/PhysRevA.61.052511

Publication date:

2000

Document Version

Publisher's PDF, also known as Version of record

Link back to DTU Orbit

Citation (APA):

Vitos, L., Johansson, B., Kollár, J., \& Skriver, H. L. (2000). Local kinetic-energy density of the Airy gas. Physical Review A, 61(5), 052511. https://doi.org/10.1103/PhysRevA.61.052511

\section{General rights}

Copyright and moral rights for the publications made accessible in the public portal are retained by the authors and/or other copyright owners and it is a condition of accessing publications that users recognise and abide by the legal requirements associated with these rights.

- Users may download and print one copy of any publication from the public portal for the purpose of private study or research.

- You may not further distribute the material or use it for any profit-making activity or commercial gain

- You may freely distribute the URL identifying the publication in the public portal

If you believe that this document breaches copyright please contact us providing details, and we will remove access to the work immediately and investigate your claim 


\title{
Local kinetic-energy density of the Airy gas
}

\author{
L. Vitos and B. Johansson \\ Condensed Matter Theory Group, Physics Department, Uppsala University, S-75121 Uppsala, Sweden
}

J. Kollár

Research Institute for Solid State Physics, H-1525 Budapest, P.O. Box 49, Hungary

H. L. Skriver

Center for Atomic-scale Materials Physics and Department of Physics, Technical University of Denmark, DK-2800 Lyngby, Denmark

(Received 30 November 1999; published 13 April 2000)

\begin{abstract}
The Airy gas model is used to derive an expression for the local kinetic energy in the linear potential approximation. The expression contains an explicit Laplacian term $\frac{2}{5}\left(\hbar^{2} / 2 m\right) \nabla_{\mu}^{2}(\mathbf{r})$ that, according to jellium surface calculations, must be a universal feature of any accurate local description. Applied to the noble gases the expression reduces the errors by a factor of 50 over previous results obtained by the linear potential approximation.

PACS number(s): 31.15.Ew, 71.15.Mb
\end{abstract}

During the last two decades density-functional theory [1] has played a decisive role in the calculation of physical properties of atoms, molecules, and solids. In this context it is important to note that all available ground-state energy density functionals lead to deficient results on account of an inappropriate representation of the kinetic-energy contribution. This deficiency may, of course, be overcome by applying the approach of Kohn and Sham [2] as it is in most density-functional calculations. The appoach, however, is based on an implicit functional that requires a complete selfconsistent solution to the Kohn-Sham equations. As a result, the determination of the kinetic-energy contribution becomes the time-limiting step in density-functional calculations. An explicit kinetic-energy functional is therefore of crucial importance in future applications of density functional theory. Furthermore, the knowledge of a kinetic energy functional for the noninteracting particles has become one of the primary aims within density-functional theory as formulated within the metageneralized gradient approximation to the exchange-correlation density functional [3].

Starting from the homogeneous electron gas including, e.g., gradient corrections, there have been many attempts to develop explicit kinetic-energy functionals [4]. In general these functionals have been developed and tested for use in atomic calculations, and they have proven to be inadequate in the treatment of, for instance, surfaces of solids [5]. Of particular relevance for the present purpose is the explicit kinetic-energy functional derived in the pioneering work by Baltin [6] on the basis of the Wigner-Kirkwood approach [4] and the linear potential approximation. It has the form

$$
t_{s}^{l i n}(\mathbf{r})=t_{s}^{T F}(\mathbf{r}) f\left(\frac{|\nabla n(\mathbf{r})|^{1 / 2}}{n(\mathbf{r})^{2 / 3}}\right),
$$

where $t_{s}^{T F}$ is the Thomas-Fermi kinetic-energy density, and the function $f$ of the scaled gradient is tabulated in Ref. [6]. The functional was subsequently applied in calculations of the kinetic energy of the noble gases, and it was found to overestimate the Hartree-Fock results by as much as a factor of 2 [7]. As a result of this failure further developments of the approach appear to have been abandoned.

Recently, Kohn and Mattson [8] put forward the concept of the edge electron gas as an appropriate starting point for the treatment of systems with edge regions. The simplest realization of the edge electron gas is the Airy gas model, and in the present paper we use this model to derive an explicit expression for the kinetic-energy density. Since the Airy gas model is based on the linear potential approximation, the resulting expression is equal to that obtained by Baltin [6] except for the fact that we isolate an explicit Laplacian term of the form

$$
\alpha \frac{\hbar^{2}}{2 m} \nabla^{2} n(\mathbf{r}), \quad \text { where } \alpha=\frac{2}{5},
$$

which we exclude when the functional is inverted to obtain an explicit expression for the kinetic-energy density in terms of the scaled gradient. That is, we write the kinetic-energy density in the form of Eq. (1), but with a different $f$, plus Laplacian (2). Since the contribution from the Laplacian to the total kinetic energy of a confined system must vanish the present functional yields kinetic energies which differ considerably from those obtained by a functional in which also the Laplacian is expanded in terms of the scaled gradient. As a result, the error in the kinetic energy of the rare gases is reduced in the present approach by approximately a factor of 50 relative to previous calculations by the linear potential approximation [7].

The starting point for the derivation of the Airy gas kinetic-energy functional is the potential

$$
v_{e f f}(z)= \begin{cases}\infty & \text { for } z \leqslant-L \\ F z & \text { for }-L<z<\infty\end{cases}
$$


which is linear in $z$, independent of $x$ and $y$, and has a hard wall at $-L$ far from the electronic edge at $z=0$. The slope of the effective potential $F=d V_{e f f} / d z$ leads to a characteristic length scale

$$
l \equiv\left(\frac{\hbar^{2}}{2 m F}\right)^{1 / 3}
$$

and the electron and kinetic-energy densities are then given by

$$
\begin{gathered}
n(z)=l^{-3} n(\zeta), \\
t_{s}(z)=\frac{\hbar^{2}}{2 m} l^{-5} t(\zeta),
\end{gathered}
$$

where $\zeta=z / l, t_{s}=\left(\hbar^{2} / 2 m\right) \Sigma^{o c c}(\nabla \psi)^{2}$, and $\psi$ are the KohnSham orbitals. Further,

$$
\begin{aligned}
n(\zeta) & =\frac{1}{2 \pi} \int_{0}^{\infty} \operatorname{Ai}^{2}\left(\zeta+\zeta^{\prime}\right) \zeta^{\prime} d \zeta^{\prime} \\
t_{s}(\zeta)= & \frac{1}{2 \pi} \int_{0}^{\infty}\left[\frac{d}{d \zeta} \operatorname{Ai}\left(\zeta+\zeta^{\prime}\right)\right]^{2} \zeta^{\prime} d \zeta^{\prime}+\frac{1}{4 \pi} \\
& \times \int_{0}^{\infty} \operatorname{Ai}^{2}\left(\zeta+\zeta^{\prime}\right) \zeta^{\prime 2} d \zeta^{\prime}
\end{aligned}
$$

The properties of the Airy function and its derivatives may be used to transform the kinetic energy density function (8) into

$$
t_{s}(\zeta)=-\frac{3}{5} \zeta n(\zeta)+\frac{2}{5} n^{\prime \prime}(\zeta)
$$

which upon application of the scalings of the $i$ th derivative

$$
n^{(i)}(z)=l^{-i-3} n^{(i)}(\zeta), \quad i=0,1,2, \ldots,
$$

characteristic of the Airy gas model, leads to the local Airy gas (LAG) expression for the kinetic-energy density of the real electron gas:

$$
t_{s}^{L A G}(z)=-\frac{3}{5} n(z)\left[\frac{\hbar^{2}}{2 m} \frac{z}{l^{3}}\right]+\frac{2}{5} \frac{\hbar^{2}}{2 m} n^{\prime \prime}(z) .
$$

According to Eq. (3) and (4), the term in the square bracket on the right-hand side of Eq. (11) is equal to $v_{e f f}(z)$, and the kinetic-energy density then reads

$$
t_{s}^{L A G}(z)=-\frac{3}{5} n(z) v_{e f f}(z)+\frac{2}{5} \frac{\hbar^{2}}{2 m} n^{\prime \prime}(z) .
$$

Alternatively, one may insert the Thomas-Fermi kineticenergy density

$$
t_{s}^{T F}(z)=\frac{3}{5} \frac{\hbar^{2}}{2 m}\left(3 \pi^{2}\right)^{2 / 3} n^{5 / 3}(z)
$$

into the first term of Eq. (11) to obtain

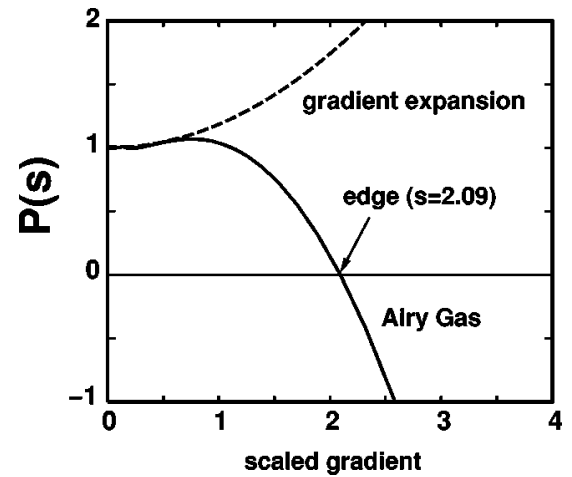

FIG. 1. The scaling function $P(s)$ of Eq. (14) obtained for the Airy gas and the second-order gradient expansions.

$$
t_{s}^{L A G}(z)=t_{s}^{T F}(z) P(s(z))+\frac{2}{5} \frac{\hbar^{2}}{2 m} n^{\prime \prime}(z),
$$

where the scaled gradient

$$
s[n(z)] \equiv \frac{|\nabla n(z)|}{2\left(3 \pi^{2}\right)^{1 / 3} n(z)^{4 / 3}}=\frac{n^{\prime}(\zeta)}{2\left(3 \pi^{2}\right)^{1 / 3} n(\zeta)^{4 / 3}} \equiv s(\zeta),
$$

on account of scaling relations (10), is conserved when going from the real electron gas to the Airy gas. Finally, we have used the properties of the Airy function to derive an explicit expression for $z / l^{3}$ in terms of the density and its derivative. We thereby find the expression

$$
\begin{aligned}
t_{s}(z)= & \frac{3}{5} \frac{\hbar^{2}}{2 m} n(z) \frac{n^{\prime \prime \prime}(z)}{4 n^{\prime \prime}(z)} \frac{3 n(z) n^{\prime \prime \prime}(z)-2 n^{\prime}(z) n^{\prime \prime}(z)}{2\left[n^{\prime}(z)\right]^{2}-3 n(z) n^{\prime \prime}(z)} \\
& +\frac{2}{5} \frac{\hbar^{2}}{2 m} n^{\prime \prime}(z)
\end{aligned}
$$

for the kinetic-energy density of the Airy gas which avoids the numerical inversion of the scaling function. However, this explicit expression is only useful in cases where the real potential is close to the linear form assumed in the Airy gas model. Note that, for an Airy gas, the three expressions (12), (14), and (16) are, of course, equivalent. However, in the application to real systems Eq. (12) is the most accurate and Eq. (16) the least accurate of the three forms.

The scaling function $P(s)$ which appears in Eq. (14) may be calculated in the Airy gas model from

$$
P(\zeta)=\frac{-\zeta}{\left(3 \pi^{2}\right)^{2 / 3} n(\zeta)^{2 / 3}}
$$

and $s(\zeta)$ defined in Eq. (15). In Fig. 1 we have plotted $(P(\zeta), s(\zeta))$ and this function provides the connection between the real system and the Airy gas through the local scaled gradient $s=s[n(z)]$ which is conserved in the transformation to the Airy gas model. The inversion procedure is the same as that used by Baltin [6], except that here it is applied only to the first term $t_{s}^{T F}(z) P(s(z))$ in the kinetic- 


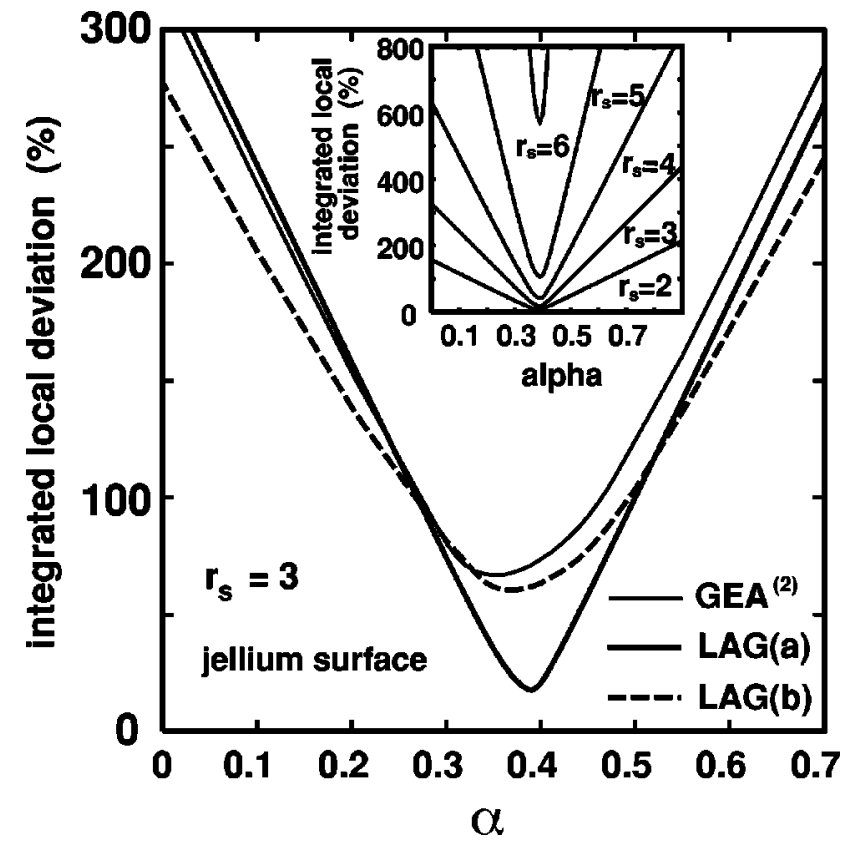

FIG. 2. The integrated local errors [Eq. (18)], of the kineticenergy densities for a self-consistent jellium surface $\left(r_{s}=3\right)$ as functions of the prefactor $\alpha$ of the Laplacian term. In the figure GEA $^{(2)}$ refers to the second order gradient expansion functional $t_{s}^{a}$ $=t_{T F}+\left(\hbar^{2} / 2 m\right) \frac{1}{9} t_{W}$, where $t_{W}$ is the von Weizsäcker functional, LAG(a) refers to $t_{s}^{a}=-\frac{3}{5} n v_{e f f}$ [cf. Eq. (12)], and LAG(b) refers to $t_{s}^{a}(z)=t_{s}^{\text {lin, } \alpha}(z)$, where $t_{s}^{\text {lin, } \alpha}(z)$ reduces to Eq. (1) for $\alpha=0.0$ and to $t_{s}^{T F}(z) P(s)$ of Eq. (14) for $\alpha=0.4$. In the inset the integrated local errors of LAG(b) for physically interesting density range $r_{s}=2-6$ are shown.

energy density expression while the Laplacian term is kept unchanged. Below we shall justify this approach.

A comparison of the kinetic energy functionals available in the literature shows that in most cases a Laplacian term of the form of Eq. (2) with varying prefactors $\alpha$ is included; see, e.g., Ref. [4]. [Here we do not refer to $\frac{1}{2}\left(\hbar^{2} / 2 m\right) \nabla^{2} n(\mathbf{r})$, which is the trivial difference between the two natural definitions of the local kinetic-energy density.] In want of a theoretical principle by which a unique value of the prefactor may be found, we have used the jellium model of a metal surface [9] for which accurate numerical solutions may be found to determine $\alpha$. That is, we have calculated the local difference between the exact Kohn-Sham kinetic-energy density $t_{s}^{K S}(z)=\left(\hbar^{2} / 2 m\right) \sum^{o c c}(\nabla \psi)^{2}$ for a jellium surface of intermediate density $r_{s}=3.0$, and that obtained by a number of kinetic-energy functionals including Laplacian terms with a range of prefactors, i.e.,

$$
\delta(\alpha)=\frac{1}{\gamma_{s}} \int\left|t_{s}^{a}(z)+\alpha \frac{\hbar^{2}}{2 m} n^{\prime \prime}(z)-t_{s}^{K S}(z)\right| d z
$$

where $t_{s}^{a}(z)$ is the "Thomas-Fermi part" of the actual kinetic-energy density, e.g., $t_{s}^{T F}(z) P(s(z))$, and $\gamma_{s}$ the total Kohn-Sham surface kinetic energy.

The results of the jellium calculations shown in Fig. 2 point to $\alpha=0.4$ as a unique value, where the asymptotic

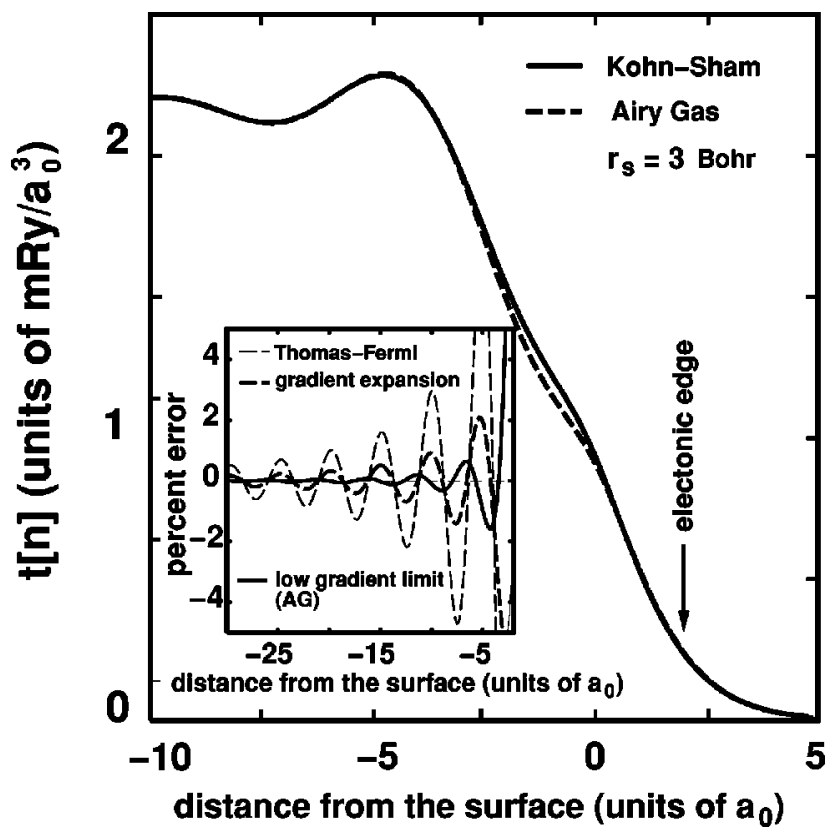

FIG. 3. The kinetic-energy densities for a self-consistent jellium surface $\left(r_{s}=3\right)$ as a function of the distance from the surface (in units of Bohr radius, $a_{0}$ ) obtained from the Airy gas expression [Eq. (12)] compared to the exact Kohn-sham results. It is seen that the airy gas expression [Eq. (12)] is almost exact for $x<-3$ and $x>0$. In the inset are shown errors in percent for the zeroth (Thomas-Fermi), the second-order gradient expansion, and the low gradient limit of Eq. (14).

linear behavior of all three functionals included in the figure extrapolate to zero error. It is further seen that at $\alpha=0.4$ the Airy gas functional (12) provides the best overall description of the kinetic-energy density while Baltin's functional, i.e., $\alpha=0$, leads to a considerable error in the local description. Since $\alpha=0.4$ is also the value found in the real-space derivation of the Airy gas functional we suggest that a Laplacian with the prefactor $\frac{2}{5}$ must be a universal feature of any local kinetic energy functional. Note that there is no a priori reason why the kinetic-energy density calculated from the Kohn-Sham orbitals for a jellium surface should correspond exactly to the prefactor $\frac{2}{5}$ for the Laplacian term.

The accuracy of the Airy gas functional (12) is demonstrated in Fig. 3, where we show the kinetic-energy density

TABLE I. The difference (in percent) in the atomic kinetic energies evaluated from the self-consistent LDA [11] Kohn-Sham densities relative to the exact Kohn-Sham results. GEA ${ }^{(0)}, \mathrm{GEA}^{(2)}$, LAG, and LLP label results obtained using the Thomas-Fermi approximation, the second-order gradient expansion [4], the Local Airy gas approximation, [10] and the locally linear potential approximation $[6,7]$.

\begin{tabular}{ccrrr}
\hline \hline Atom & GEA $^{(0)}$ & GEA $^{(2)}$ & LAG & LLP \\
\hline $\mathrm{Ne}$ & -8.6 & -0.8 & -2.3 & 68.3 \\
$\mathrm{Ar}$ & -6.8 & -0.4 & -1.2 & 53.8 \\
$\mathrm{Kr}$ & -5.1 & 0.2 & -0.1 & 38.9 \\
$\mathrm{Xe}$ & -3.3 & 1.6 & 1.4 & 31.9 \\
\hline \hline
\end{tabular}


of the jellium surface model calculated in four different approaches. The only significant deviation between the Airy gas functional (12), and the exact Kohn-Sham result is seen to occur just below the jellium edge where the effective potential has a large positive curvature which cannot be captured by the linear potential approximation. In contrast, the Thomas-Fermi and second-order gradient functionals have significant errors even far from the jellium edge. It is clear that an accurate local description also leads to an accurate total kinetic energy. In fact, the Airy gas functional and the second-order gradient expansion functional yield total kinetic energies for the jellium surface which, however, are of the same order of magnitude because the oscillations in the local gradient expansion lead to a cancellations of errors.

Since the contribution from a Laplacian term on account of Green's theorem vanishes for a confined system, it follows that only the Thomas-Fermi part of the kinetic-energy density (14) is important in calculations of the total kinetic energy. Consequently, one should not expand the Laplacian of the density in terms of the scaled gradient because such an expansion will lead to a nonvanishing contribution to the total kinetic energy from the expanded Laplacian term. That this is in fact the case is clearly shown by the results of the atomic calculations for the noble gases presented in Table I. Here we find that the Airy gas functional [10] has the accuracy of the second-order gradient expansion, as in the case of the jellium surface calculations, in contrast to the previous calculations by the linear potential approximation [6,7] which show significant errors. We conclude, that the Airy gas model and the equivalent linear potential approximation may in fact form an appropriate and well-defined starting point for density-functional theories of the kinetic energy density of the inhomogeneous electron gas.

We gratefully acknowledge interesting and fruitful discussions with Professor Walter Kohn. Thanks are also due to Doctor Karsten Jacobsen and Doctor Andrei Ruban for their valuable comments. L.V. and B.J. are grateful to the Swedish Natural Science Research Council and the Swedish Foundation for Strategic Research for financial support. Part of this work was supported by the research Project No. OTKA 23390 of the Hungarian Scientific Research Fund. The Center for Atomic-scale Materials Physics is sponsored by the Danish National Research Foundation.
[1] P. Hohenberg and W. Kohn, Phys. Rev. B 136, 864 (1964).

[2] W. Kohn and L.J. Sham, Phys. Rev. A 140, 1133 (1965).

[3] J. P. Perdew, S. Kurth, A. Zupan, and P. Blaha, Phys. Rev. Lett. 82, 2544 (1999).

[4] R. M. Dreizler and E. K. U. Gross, in Density Functional Theory (Springer-Verlag, Berlin, 1990), and references therein.

[5] L. Vitos, H. L. Skriver, and J. Kollár, Phys. Rev. B 57, 12611 (1998).

[6] R. Baltin, Z. Naturforsch. Teil A 27, 1176 (1972).

[7] S. K. Ghosh and L. C. Balbás, J. Chem. Phys. 83, 5778 (1985).
[8] W. Kohn and A. E. Mattsson, Phys. Rev. Lett. 81, 3487 (1998).

[9] N. D. Lang and W. Kohn, Phys. Rev. B 1, 4555 (1970).

[10] In these calculations we used the Padé approximation $P(s)$ $=\left(1+a_{1} s^{2}-a_{3} s^{6}\right) /\left(1+a_{2} s^{2}+a_{3} s^{4}\right)$ for the scaling function. For $a_{1}=0.8944, a_{2}=0.6511$, and $a_{3}=0.0431$ the relative error between the exact result and the parametrized form for $s \leqslant 10$ was less than $0.5 \%$.

[11] J. Perdew and Y. Wang, Phys. Rev. B 45, 13244 (1992). 